\title{
Effects of Knee Extensor Muscle Fatigue on Gait Ability in Patients with Chronic Stroke
}

\author{
Byung-Il Yang ${ }^{1}$, Sang-Mi Joung ${ }^{*}$ \\ ${ }^{1}$ Dept. of Physical Therapy, Sangji University, Gangwon-do, Republic of Korea \\ ${ }^{* 2}$ Dept. of Occupational Therapy, Sangji Youngseo University, Gangwon-do, Republic of Korea
}

Purpose The purpose of this study were how to effect gait ability to chronic stroke patients who were taken knee extensor fatigue and were understood and offered the way of effective therapy to stroke patients in clinical trials. Methods The subject of this study were 16 chronic stroke patients (9 males, 8 females), and 16 healthy volunteers(5 males, 11 females) and their average duration of symptoms were 4.9 years at $\mathrm{S}$ hospital in Sungnam city. Stroke patient group age was 55.67, health group age was 59.13. Both stroke patient group and healthy group were measured using maximal voluntary isometric contraction(MVIC) for leading knee extensor fatigue. Isometric contraction was maintained for 10 seconds. And each isometric contraction was taken rest 5 seconds one time for inducing muscle fatigue. The end point was measured decreasing 3 times continuously which the value of MVIC was less $50 \%$, and then the number of knee extensor to induce muscle fatigue gait ability were measured after taking knee extensor fatigue. Results There was a significantly differences that Stroke patients group was shown that the paretic side knee extensor fatigue was represented faster than health control group ( $<<0.05)$. The gait ability were represented drastically decreased knee extensor fatigue in Stroke patients group's non paretic side. Conclusion Therefore, localized muscle fatigue by gaiting abnormal gait pattern to chronic stroke patient was affected the disability of gait capacity. And abnormal gait pattern was brought secondly increasing number of falling down. These factors were considered to stroke rehabilitation.

Key words Knee extensor, Paretic, Nonparetic, Muscle fatigue, Stroke

Corresponding author Sang Mi Joung (otjsm@daum.net)

Received date 09 January 2019

Revised date 30 January 2019

Accepted date 13 February 2019

\section{INTRODUCTION}

Stroke is an acquired disease that causes considerable disability for more than a few months despite active rehabilitation. It is a classic cause of paraplegia. Hemiplegic patients have damaged posture control because of strokes. Motion, sensory, and high central cognitive defects due to a stroke cause loss of function. Most patients have limitations throughout their community activities due to independent walking disorders, causing secondary physical disability problems.

Secondary physical disorders include asymmetrical

http:dx.doi.org/10.17817/2019.01.30.111353 posture and abnormal walking patterns, which can also lead to pain, muscle weakness, stiffness, sensory information disturbance, and abnormal strain.

The ideal walk for normal people should be a symmetrical weight, bearing on the body with a continuous, repetitive, symmetrical motion, and a symmetrical walking cycle and walking speed. The cycle, velocity, step length, and stride length are important for stroke patients. However, the characteristic walk of a hemiparetic patient due to stroke shows asymmetric weight load, slow walking cycles, and walking speed. These asymmetrical support times affect walking speed and walking cycles. ${ }^{1)}$ The walking speed of a stroke patient is related to the differences in step 
length, step width, swing phase, and stance phase. As a result, walking in hemiplegic patients results in a decrease in the time of the paralysis side and an increase in the time of the non-paretic side swing time period. ${ }^{2)}$ In addition to this, it interferes with the selective movement control ability in gait and causes a decrease in voluntary muscle activation and muscle recruitment ability. Therefore, most hemiplegic patients support the weight on the paretic side because of unstable standing and abnormal gait. ${ }^{1-2)}$ Therefore, according to the asymmetric gait pattern, patients have a longer time in the stance phase of the paretic side, resulting in inefficient energy consumption and slow walking. It is reported that muscle fatigue and tension are reduced due to excessive weight support of the non-paretic side. ${ }^{2-4)}$

Muscular fatigue is the gradual decrease in the ability of the acute muscles to exert force because of exercise; there is decrease in muscle strength and mental function as well as lack of exercise." Skeletal muscle fibers are classified according to the rate of contraction reaction to electrical stimulation. They are classified into slow twitch oxidative fibers (I type) and fast twitch glycolytic fibers (IIb type). Fast twitch glycolytic fibers have a fast muscle contraction rate and tire easily. ${ }^{6-7)}$ Muscle fatigue is known to reduce motor control capacity. ${ }^{8)}$ Reduced motor control capacity may be caused by decreased ability to maintain muscle strength due to fatigue. As a result, muscle fatigue causes a decrease in proprioceptive sensation. $^{9-10)}$ Also, Muscle fatigue cause impair the proprioceptive and kinesthetic properties of joints by increasing the threshold of muscle spindle discharge. Recent studies have reported that vestibular information, visual information, and neck somatosensory are also closely related to postural control. As a result, incorrect somatosensory information due to muscle fatigue causes fluctuations in postural control. ${ }^{9-11}$ ) Studies on muscle fibers and position senses were previously conducted. ${ }^{12)}$

Parijat and Lockhart(2008) made a study on young subjects and reported that localized muscle fatigue of the proximal knee extensor negatively affected the position of the knee joint, and it caused a change in the gait pattern by interfering with the movement of the joint. ${ }^{13)}$

There are many studies that have focused on gender, age, and focal localized muscle fatigue for normal subjects, but there are a lack of studies on the effects of fatigue on the paretic and the non-paretic side of the knee extensor muscles in stroke patients. The purpose of this study was to investigate the effect of the knee extensor on the gait pattern in hemiplegic patients and to provide understanding and improved therapeutic efficacy of stroke patients in clinical practice. This study followed the ethical principles of the Declaration of Helsinki, and all patients gave written informed consents before participating in this study. We approved the approval of the Institutional Life Research Ethics Committee of Yongin University (2-1040966-AB-N-01-20-1611-HSR061-8).

\section{Material and Methods}

\section{Subjects}

In this study, 16 stroke patients and 16 control subjects participated in S hospital in Seongnam City. The study subjects were 32 persons who agreed to participate in this study.

The inclusion criteria for the stroke patient group were as follows: (1) Sub acute stage patients (6 months to 1 year after onset), (2) No damage to position sense, (3) No cognitive deficits (MMSE-K score of $\geq 24$ points), (4) No joint deformity and orthopedic disease, (5) Understanding and informed consent to this study

\section{Procedure}

This study was performed on the stroke paretic side, the non-paretic side, the normal control group dominant side, and the normal control group non-dominant side. The order was randomly assigned.

This study was designed to evaluate the maximum voluntary isometric contraction of the knee extensor using the Primus dynamometer (Baltimore Therapeutic Equipment, USA, 2006). The subjects were seated on a Primus dynamometer with $10^{\circ}$ backward sitting, sit- 
ting with the hip joint at $80^{\circ}$ and the knee joint at $60^{\circ}\left(0^{\circ}=\right.$ full extension $)$ in an upright position. During maximal voluntary isometric contraction, the upper body and thigh were immobilized in a velcro. The knee joint was aligned with the axis to perform knee flexion at $\left.60^{\circ}{ }^{14}\right)$ The maximum isometric contraction force measurement was performed by isometric contractions of the knee extensor four times for five seconds to determine the maximum voluntary contraction force and to provide auditory and visual feedback for the subject's continued efforts in the measurement. In order to eliminate the effect of muscle fatigue during the measurement, a 2-minute rest period was given for each contraction. ${ }^{15)}$ The measured values were recorded using Newton-meters. When the maximum value exceeded $10 \%$ in four measurements, the remaining values were excluded and the average value was selected as the maximum voluntary isometric contraction value.

\section{Muscle fatigue measurement}

The subjects were in the same posture as for the maximum voluntary isometric contraction force measurements. The isometric contraction of the knee extensor was continuously sustained for ten seconds, and the maximum isometric contraction was followed by a 5 -second rest period to induce muscle fatigue. The end point of the isometric contraction was until the maximum isometric contraction decreased to less than $50 \%$ at three consecutive times. Gait ability tests were done immediately after muscle fatigue set in [14-15]. The subjects used a wheelchair to minimize the recovery of the knee extensor after muscle fatigue and to ensure the safety of the subject. ${ }^{16)}$

\section{Gait ability measurement}

GaitrRite (CIR system Inc, USA, 2008) was used for the gait ability test. After the patient walked in front of the gait board, he was guided to walk at the most comfortable walking speed by a verbal sign, "Walk comfortably", on the gait board. The GaitRite is equipped with a sensor on an electronic gait plate with a length of $3 \mathrm{~m}$, a width of $61 \mathrm{~cm}$, and a height of 0.6 $\mathrm{cm}$. The time and spatial variables during walking on the mat was evaluated using a computer.

Information on collected temporal and spatial variables was processed with GAITRite GOLD, Version 3.2b (CIR system Inc, USA, 2007) software.

\section{Data analysis}

In this study, SPSS 20.0 program for Windows was used. Differences between pre- and post-test were analyzed by paired t-test and one-way ANOVA was performed to compare differences between the groups. When there were significant differences, Tukey's Honest Significant Difference test was performed. Statistically significant levels were $=$ to 0.05 .

\section{Results}

The subjects were 16 chronic stroke patients $(9$ males and 8 females) and 16 normal peoples $(5$ males and 11 females). In the stroke patients, four was tested on the right side and 12 on the left side. The mean duration of the disease was 4.9 years. The mean age was 55.67 years in the patient group and 59.13 years in the control group. According to this study, the score of the Functional Ambulation Profile was reduced from the initial assessment of 74.31 points to 69.69 points after fatigue on the paretic side of the patient's knee. For the non-paretic side fatigue, it decreased to 69.75 points $(\mathrm{p}<.01)$ (Table 1$)$. In the normal control group, the initial evaluation of 96.75 points was reduced to 94.56 points after non-dominant side fatigue. In addition to this, after fatigue on the dominant side, the initial evaluation of 94.81 points decreased by 1.94 points. These results showed statistically significant differences in the normal controls $(\mathrm{p}<.01) \quad($ Table2). After non-paretic side knee extensor fatigue, the velocities decreased significantly from 68.10 to 62.29 , indicating a significant difference $(p<.05)$. There was no significant difference after paretic side knee extensor fatigue (Table 1). In the normal control group, there was no significant difference between before and after fatigue. 
Table 1. The gait ability in patients before and after fatigue knee extensor

\begin{tabular}{|c|c|c|c|c|c|c|c|c|}
\hline & Pre-fatigue & $\begin{array}{l}\text { Non dominant } \\
\text { Post-fatigue }\end{array}$ & & & Pre-fatigue & $\begin{array}{l}\text { Dominant } \\
\text { Post-fatigue }\end{array}$ & & \\
\hline & $\mathrm{M} \pm \mathrm{SE}$ & $\mathrm{M} \pm \mathrm{SE}$ & $\mathrm{t}$ & $\mathrm{p}$ & $\mathrm{M} \pm \mathrm{SE}$ & $\mathrm{M} \pm \mathrm{SE}$ & $\mathrm{t}$ & p \\
\hline $\begin{array}{l}\text { Velocity } \\
(\mathrm{cm} / \mathrm{sec})\end{array}$ & $68.10 \pm 6.9$ & $63.44 \pm 6.34$ & 1.721 & .106 & $68.10 \pm 6.9$ & $62.29 \pm 6.57$ & 2.861 & $.012^{*}$ \\
\hline $\begin{array}{l}\text { Cadence } \\
\text { (step/min) }\end{array}$ & $92.48 \pm 4.05$ & $91.83 \pm 3.81$ & .386 & .705 & $92.48 \pm 4.05$ & $89.69 \pm 2.92$ & 2.130 & .050 \\
\hline $\begin{array}{c}\text { Functional Amb. } \\
\text { (score) }\end{array}$ & $74.31 \pm 4.59$ & $69.69 \pm 4.42$ & 2.432 & $.028^{*}$ & $74.31 \pm 4.59$ & $69.75 \pm 4.61$ & 3.047 & $.008^{* *}$ \\
\hline
\end{tabular}

Table 2. The gait ability in healthy control before and after fatigue knee extensor

\begin{tabular}{|c|c|c|c|c|c|c|c|c|}
\hline & Pre-fatigue & $\begin{array}{l}\text { Non dominant } \\
\text { Post-fatigue }\end{array}$ & & & Pre-fatigue & $\begin{array}{c}\text { Dominant } \\
\text { Post-fatigue }\end{array}$ & & \\
\hline & $\mathrm{M} \pm \mathrm{SE}$ & $\mathrm{M} \pm \mathrm{SE}$ & $\mathrm{t}$ & $\mathrm{p}$ & $\mathrm{M} \pm \mathrm{SE}$ & $\mathrm{M} \pm \mathrm{SE}$ & $\mathrm{t}$ & $p$ \\
\hline $\begin{array}{l}\text { Velocity } \\
(\mathrm{cm} / \mathrm{sec})\end{array}$ & $107.34 \pm 3.01$ & $101.84 \pm 3.18$ & 1.832 & 0.087 & $107.34 \pm 3.01$ & $106.93 \pm 4.08$ & 0.15 & 0.883 \\
\hline $\begin{array}{c}\text { Cadence } \\
\text { (step/min) }\end{array}$ & $113.46 \pm 2.45$ & $110.66 \pm 2.92$ & 1.26 & 0.227 & $113.46 \pm 2.45$ & $112.63 \pm 2.29$ & 0.428 & 0.675 \\
\hline $\begin{array}{l}\text { Functional Amb. } \\
\text { (score) }\end{array}$ & $96.75 \pm 0.74$ & $94.56 \pm 0.63$ & 4.68 & $0.000^{* * *}$ & $96.75 \pm 0.74$ & $94.81 \pm 0.68$ & 7.766 & $0.000^{* *}$ \\
\hline
\end{tabular}

\section{Discussion}

The purpose of this study was to investigate the effects of knee extensor fatigue on the gait ability of chronic stroke patients, particularly hemiplegic patients. Fatigue of the knee extensor was performed using a Primus dynamometer (Baltimore Therapeutic Equipment, USA, 2006), which is a frequently used induction method for voluntary fatigue induction tests. ${ }^{17)}$ Stroke patients reported muscle weakness on the contralateral limb due to cerebral cortical damage. The maximum voluntary isometric contraction of the knee extensor significantly decreased the torque value in both paralyzed and non-paralyzed subjects compared to normal subjects. ${ }^{14,}{ }^{18)}$

This suggests that voluntary activation failure of the knee extensor occurs in both sides, confirming the theory that neurological damage directly affects skeletal muscle. ${ }^{14)}$ Voluntary activation failure may be due to a motor unit recruitment failure, or it may be due to a decrease in the rate of firing of the active units. ${ }^{16)}$ The skeletal muscles of hemiplegic patients due to stroke have slow contraction and relaxation and the paralyzed limb still moved slower than normal after 6 months. In addition to this, stroke patients have decreased oxidative metabolism, resulting in decreased exercise capacity and muscle endurance. Resistance to muscle fatigue weakens functional mobility. ${ }^{14)}$ This is because stroke patients take two to three times longer than normal to produce strength when walking. ${ }^{4}$ Stroke patients also cannot maintain effective walking speeds on their own. As a result of this, high energy consumption and poor muscle endurance affect functional performance ability. Dean et al. (2001) described endurance shortages in $14 \mathrm{pa}-$ tients with chronic stroke, which suggests that stroke patients with similar body characteristics can walk only about $50 \%$ of the normal walking distance. In stroke patients, $80 \%$ of body weight was supported on the non-paretic side leg, and when standing, the paretic side lower limb supported less than 50\% of the total body weight. ${ }^{3)}$ The gait characteristics of these stroke patients were twice as long as in the normal control group in the double support phase, 
which required more time for the patients to maintain balance. Thus, the short stance phase time of the paralyzed leg during gait is because it causes muscle fatigue of the non-paretic lower limb muscle. These factors can lead to more balance and walking disorders. Therefore, asymmetrical gait characteristics of stroke patients lead to overuse of the non-paretic side lower limb; this easily induces muscle fatigue. In this study, we found that the non-paretic knee extensor muscle fatigue showed a decrease in walking ability as compared to the paretic side knee extensor muscle fatigue (Table 1).

According to Dean Order, the walking support time in the single support phase of the patients with hemiplegia decreased in both the paretic side and non-paretic side (biceps femoris and femoral rectus, $\mathrm{p}<.05)$ as compared with the normal control group. Co-contraction time of biceps femoris and rectus femoris increased significantly in stroke patients $(p<.05) .{ }^{19)}$ As a result of this, stroke patients used more double support time than single support time during walking and the propulsive forces on the paretic side and the non-paretic side reduction caused abnormal gait. ${ }^{19)}$ The results of this study showed that hemiplegic patients had greater gait disturbance on the non-paretic knee extensor muscle fatigue because of the longer time required for double support phase as compared to normal patients (Table 1). The purpose of this study was to investigate the effect of artificial local knee extensor fatigue on walking in hemiplegic patients. This is not a natural fatigue of everyday life, so it is difficult to generalize research results. The results of this study were limited because all the chronic stroke patients were not grouped into recovery stages (acute, subacute, and chronic). In addition, this study was limited to interpretation of patients with everting stroke patients because it was performed in patients with chronic stroke without being grouped into recovery stages (acute, subacute, and chronic).

After having a stroke, a patient has abnormal posture control and gait disturbances due to impaired motor and sensory function. This leads to increased postural sway, asymmetrical weight support, impaired ability to move weight, and persistent abnormal gait patterns. Gait training leads to localized muscle fatigue because of the non-paretic side. Especially, Fatigue at the knee extensor led to postural control impairment in the frontal plane. Therefore, localized muscle fatigue on the non-paretic side may result in decreased balance ability and gait disturbance of the patient, and abnormal gait pattern may lead to secondary risk of falls. In the future, it will be necessary to consider these factors in stroke gait learning.

\section{References}

1. Yang YR, Yen JG, Wang RY, et al. Gait outcomes after additional backward walking training in patients with stroke. A randomized controlled trial. ClinRehabil. 2005;19(3):264-37.

2. De Quervain AK, Simon SR, Leurgans S, et al. Gait pattern in the early recovery period after stroke. J Bone Joint Surg Am. 1996;78(10):1506-1514.

3. Dean CM, Richards CL, Malouin F. Walking speed over 10 meters overestimates locomotor capacity after stroke. Clin Rehabil. 2001;15:415-421.

4. Canning CG, Ada L, O'Dwyer N. Slowness to develop force contributes to weakness after stroke. Arch of Phys Med Rehabi. 1999;80:66-70.

5. Enoka RM, Duchateau J. Muscle fatique: What, why and how it influences muscle function. J Physiol. 2008;586(1):11-23.

6. Toffola ED, Sparapaglione D, Pistorio A, et al. Myoelectric manifestation of muscle change in stroke patients. 2001;82(5):661-665.

7. Gribble PA, Hertel J. Effect of lower-extremity muscle fatigue on postural control. Arch Phys Med Rehabil. 2004;85:589-592.

8. Bellew JW, Fenter PC. Control of balance differs after knee or ankle fatigue in older women. Arch Phys Med Rehabil. 2006;87:1486-1489.

9. Sharpe MH, Miles TS. "Position sense at the elbow after fatiguing contraction." Exp Brain Res. 1993;94:179-182.

10. Salavati M, Moghadam M, Ebrahimi I, et al. Changes in postural stability with fatigue of lower extremity frontal and sagittal plane movers. Gait Posture. 2007;26:214-218.

11. Vuillerme N, Pinsault N. Vestibular and neck somatosensory weighting changes with trunk extensor muscle fatigue during standing. Exp Brain Res. 2010;202(1):253-259. 
12. Barrett DS. Proprioception and function after anterior cruciate reconstruction. $\mathrm{J}$ Bone Joint Surg Br. 1991;73(5):833-837.

13. Parijat P, Lockhart TE. Effects of quadriceps fatigue on the biomechanics of gait and slip propensity. Gait Posture. 2008;28:568-573.

14. Horstman AM, Gerrits KH, Beltman MJ, et al. Intrinsic properties of the knee extensor muscles after subacute stroke. Arch Phys Med Rehail. 2010;91:123-128.

15. Jacobs C, Uhi TL, Seeley M, et al. Strength and fatigability of the dominant and nondominant hip abductors. J Athl Train. 2005;40(3):203-206.

16. Wojtys EM, Wylie BB, Huston LJ. The effects of muscle fatigue on neuromuscular function and anterior tibial translation in healthy knees. Am J Sport Med. 1996;24(5):615-621.
17. Vollestad NK. Measurement of human muscle fatigue. J Neuroscien Methods. 1997;74:219-27.

18. Newham DJ, Hsiao SF. Knee muscle isometric strength, voluntary activation and antagonist co-contraction in the first six months after stroke. Disabil Rehabil. 2001;23(9):379-86.

19. Den Otter AG, Guerts AC, Mulder T, et al. Abnormalities in the temporal patterning lower extremity muscle activity in hemiparetic gait. Gait Posture. 2007:25(3):342-52. 